\title{
Roe Scheme for Two-layer Shallow Water Equations: Application to the Strait of Gibraltar
}

\author{
M. Chakir ${ }^{1}$, D. Ouazar ${ }^{2}$ and A. Taik ${ }^{3, *}$ \\ ${ }^{1}$ Information Management and Technology, GIS Branch, New Brunswick, Department \\ of Transportation, Kings Place, 440 King Street Fredericton N-B E3B-5H8, Canada \\ ${ }^{2}$ Univ. Mohammed V, EMI, LASH, Av. Ibn Sina -B.P.765 Agdal, Rabat, Morocco \\ ${ }^{3}$ Department of Mathematics, FSTM, HassanII-Mohammedia University, Morocco
}

\begin{abstract}
The flow trough the Strait of Gibraltar could be analyzed as a problem of two-layer hydraulic exchange between the Atlantic ocean and the Mediterranean sea. The shallow water equations in both layers coupled together are an important tool to simulate this phenomenon. In this paper we perform an upwind schemes for hyperbolic equations based on the Roe approximate Riemann solver, to study the resulting model. The main goal assigned was to predict the location of the interface between the two layers. Therefore the computational results obtained are compared to previous results and experiments.
\end{abstract}

Key words: Strait of Gibraltar, Atlantic ocean layer, Mediterranean sea layer, interface between layers, shallow water model, finite volume method, Roe approximate Riemann solver

AMS subject classification: 65M12, 75M08, 76B15, 78B70

\section{Introduction}

The Strait of Gibraltar is located between the southern coast of Spain and northern coast of Morocco $\left(35^{\circ} 45^{\prime}\right.$ to $36^{\circ} 10^{\prime} \mathrm{N}$. latitude and $5^{\circ} 10^{\prime}$ to $6^{\circ} 00^{\prime} \mathrm{W}$. longitude) (see figure 1$)$. It is the single connection between the Atlantic Ocean and the Mediterranean Sea. Water flow through the strait consists of an upper layer of Atlantic water flowing eastward over a lower layer of Mediterranean water flowing westward.

\footnotetext{
*Corresponding author. E-mail: taik_ahmed@yahoo.fr
} 
The Strait has been an area of intense study in order to understand and characterize this exchange of water between the Atlantic and Mediterranean. Early studies are described in Defant (1961), while Lacombe and Richez (1982, [14]) described an investigation carried out in the 1960's over several campaigns under the aegis of the NATO subcommittee on Oceanographic Research. Those data were also used by Armi and Farmer ((1985), [2]) to interpret the hydraulic flow through the Strait and helped to motivate the Gibraltar Experiment carried out in 1985 - 1986 [La Violette et al (1986) [16]; Kinder and Bryden 1987, Armi and Farmer (1988), [3]]. Armi and Farmer used data collected as part of that experiment to examine the details of the flow within the Strait, identifying a number of hydraulic control points at the Tarifa Narrow, the Camarinal Sill, the Spartel Sill and at a location west of the Spartel Sill. A comprehensive atlas of the hydrology and currents can be found in [Lacombe and Richez (1984), [15]].

The density difference between the Atlantic and Mediterranean waters, due primarily to their salinity differences, provides the necessary stratification. So the two well defined water masses are separated by a relatively sharp interface. As proved by many experiments ([6], [9]) the system can therefore be approximated by a two-layer model. Many attempts to understand and measure the basic parameters governing the multi-layered flow in the Strait of Gibraltar have been undertaken ([4], [6], [8], [9], [14]\&[19]). On October 24, 1980 Spain and Morocco signed a scientific and technical co-operation agreement for the study of the feasibility of a fixed link Europe-Africa through the Strait of Gibraltar. Recently the solution of the rail tunnel under the Strait of Gibraltar is preferred to the suspended bridge $[12,25]$. Regarding this important project, every study and information on the subject will be benefic and helpful.

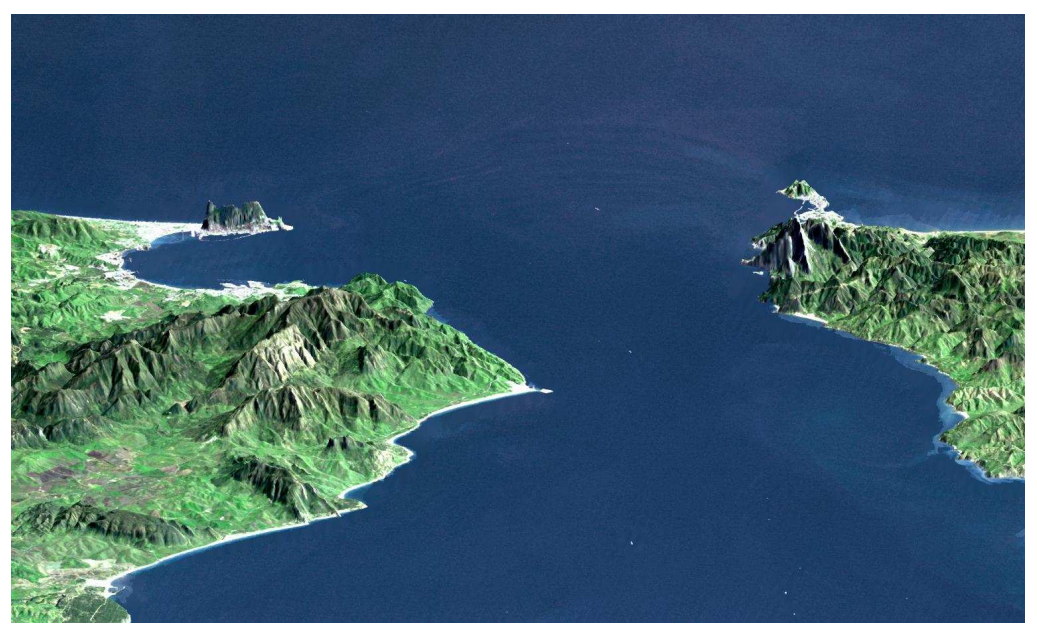

Figure 1: 3D view of the Strait of Gibraltar

Bryden and Stommel [10] developed a pure algebraic model for the sill section and they got a surprisingly good results. Ouazar et Al [22] generalized the Bryden's model along the Strait using the one dimensional approximation, neglecting the secondary effects and have developed a numerical model based on the method of characteristic (MOC) to solve the resulting shallow water equation. 
Explicit and implicit methods, also finite element method have been used to solve the shallow water equations. One feature of this set of hyperbolic equations is the formation of discontinuous solutions, which can be difficult to represent accurately. However, the development of finite volume method for hyperbolic conservation laws has been a rapidly growing area for the last decade due to its attractive features and specifically nice shock capturing ([17], [18], [26]). In this paper we use this technique to study the shallow water equations resulting from the two-layer model. To test the numerical simulation, some results are compared to the experimental data observed by Kinder in 1985 (CTD measurement [6], [8]), and to the numerical results obtained by Ouazar et Al. [22] and $[20],[21]$.

\section{Mathematical formulation}

In most circumstances, the sea surface elevations and current due to tides and storm surge can be described by the inviscid shallow water equations. The flow is assumed to be vertically well mixed with a hydrostatic pressure gradient and then taking into account the kinematic boundary conditions on the free surface. In their primitive form, the depth-integrated governing equations include a continuity equation and momentum equation in each of the $x$ and $y$ directions $[13,24]$ :

$$
\left\{\begin{array}{l}
\frac{\partial h}{\partial t}+\frac{\partial(h u)}{\partial x}+\frac{\partial(h v)}{\partial y}=0 \\
\frac{\partial(h u)}{\partial t}+\frac{\partial(u h u)}{\partial x}+g h \frac{\partial h}{\partial x}+\frac{\partial(v h u)}{\partial y}=g h S_{0 x}+f v h+\tau_{x}-\tau_{b x} \\
\frac{\partial(h v)}{\partial t}+\frac{\partial(v h v)}{\partial x}+g h \frac{\partial h}{\partial x}+\frac{\partial(u h v)}{\partial y}=g h S_{0 y}-f u h+\tau_{y}-\tau_{b y}
\end{array}\right.
$$

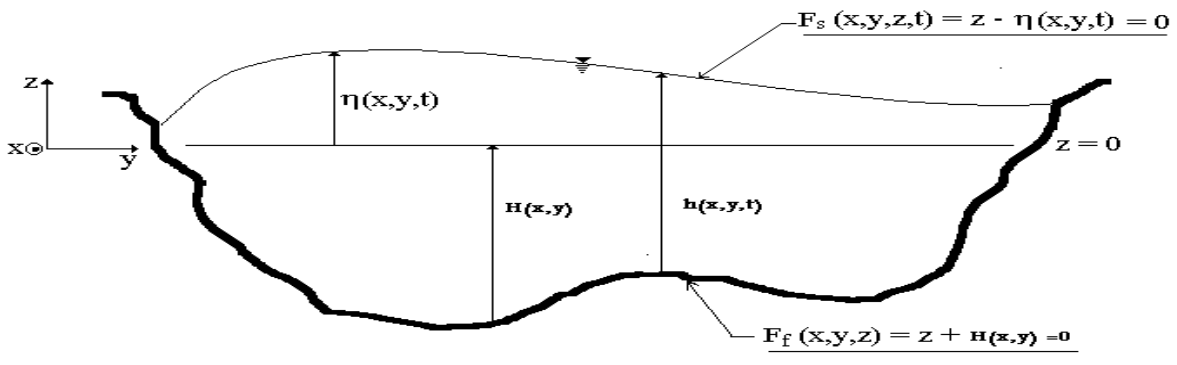

Figure 2: Description of the physical variables

where $x, y$ are the horizontal coordinates, $t$ is the time, $u=u(x, y, t)$ is the depth-averaged velocity in the $x$ direction. $v=v(x, y, t)$ is the depth-averaged velocity in the $y$ direction. $h=$ $h(x, y, t)$ is the instantaneous water depth, $\eta$ is the elevation of the free surface and $h=H+\eta$ (see figure 2). $g$ stains for the gravity acceleration, $f$ is the coriolis parameter. $S_{0 x}$ and $S_{0 y}$ are the 
bottom bed slopes defined as: $S_{0 x}=-\frac{\partial Z_{f}}{\partial x}$ and $S_{0 y}=-\frac{\partial Z_{f}}{\partial y}, Z_{f}$ being the bed elevation. $\tau_{b x}, \tau_{b y}$ correspond to the bottom friction slopes. $\tau_{x, y}$ are the wind stresses.

Suppose that we have two layers of water with densities $\rho_{1,2}$. This may be useful approximation in stratified seas. The integration process, described above (equations (2.1)), can be then performed for each layer separately, taking into account exchange of mass and momentum across the interface. In the special case that there is no mass exchange, assuming:

- The flow is quasi-horizontal, i.e. one can neglect the $y$ component.

- The density is uniform in each layer.

The following equations results:

\section{Atlantic layer (upper layer)}

$$
\left\{\begin{array}{l}
\frac{\partial h_{1}}{\partial t}+\frac{\partial\left(h_{1} u_{1}\right)}{\partial x}=0 \\
\frac{\partial\left(h_{1} u_{1}\right)}{\partial t}+\frac{\partial}{\partial x}\left(\frac{\left(h_{1} u_{1}\right)^{2}}{h_{1}}+\frac{g h_{1}^{2}}{2}\right)=-g h_{1}\left(\frac{\partial h_{2}}{\partial x}+\frac{\partial Z_{f}}{\partial x}\right)+\tau_{w}-\tau_{I}
\end{array}\right.
$$

Mediterranean layer (lower layer)

$$
\left\{\begin{array}{l}
\frac{\partial h_{2}}{\partial t}+\frac{\partial\left(h_{2} u_{2}\right)}{\partial x}=0 \\
\frac{\partial\left(h_{2} u_{2}\right)}{\partial t}+\frac{\partial}{\partial x}\left(\frac{\left(h_{2} u_{2}\right)^{2}}{h_{2}}+\frac{g h_{2}^{2}}{2}\right)=-g h_{2}\left(\frac{\rho_{1}}{\rho_{2}} \frac{\partial h_{1}}{\partial x}+\frac{\partial Z_{f}}{\partial x}\right)+\tau_{I}-\tau_{b},
\end{array}\right.
$$

in general conservative form the equations (2.2)-(2.3) can be written as the following

$$
\begin{aligned}
& \left\{\begin{array}{l}
\frac{\partial w_{1}}{\partial t}(x, t)+\frac{\partial F_{1}}{\partial x}\left(w_{1}(x, t)\right)=S_{1}\left(x, w_{1}(x, t), w_{2}(x, t)\right), \\
x \in I \subset I R, t \in[0, T] .
\end{array}\right. \\
& \left\{\begin{array}{l}
\frac{\partial w_{2}}{\partial t}(x, t)+\frac{\partial F_{2}}{\partial x}\left(w_{2}(x, t)\right)=S_{2}\left(x, w_{1}(x, t), w_{2}(x, t)\right), \\
x \in I \subset I R, t \in[0, T] .
\end{array}\right.
\end{aligned}
$$

Where $w_{i}$ is the vector of flow variables, $F_{i}$ is the cartesian components of momentum flux for each layer.

$$
w_{i}(x, t)=\left(\begin{array}{c}
h_{i}(x, t) \\
q_{i}(x, t)
\end{array}\right)=\left(\begin{array}{c}
h_{i}(x, t) \\
h_{i}(x, t) u_{i}(x, t)
\end{array}\right), F_{i}\left(w_{i}\right)=\left(\begin{array}{c}
q_{i} \\
\frac{q_{i}^{2}}{h_{i}}+\frac{g h_{i}^{2}}{2}
\end{array}\right), i=1,2
$$




$$
\begin{aligned}
& S_{1}\left(x, w_{1}, w_{2}\right)=\left(\begin{array}{c}
0 \\
-g h_{1}\left(\frac{\partial h_{2}}{\partial x}+\frac{\partial Z_{f}}{\partial x}\right)+\tau_{w}-\tau_{I}
\end{array}\right), \\
& S_{2}\left(x, w_{1}, w_{2}\right)=\left(\begin{array}{c}
0 \\
g h_{2}\left(\frac{\rho_{2}}{\rho_{1}} \frac{\partial h_{1}}{\partial x}-\frac{\partial Z_{f}}{\partial x}\right)+\tau_{I}-\tau_{b}
\end{array}\right) .
\end{aligned}
$$

Equations (2.4) and (2.5) are coupled through their source terms $S_{1}\left(x, w_{1}, w_{2}\right)$ and $S_{2}\left(x, w_{1}, w_{2}\right)$. Here $h_{i}(x, t), i=1,2$ denote respectively the depth of the Atlantic layer at point $\mathrm{x}$ and at time $\mathrm{t}$, the depth of the Mediterranean layer, $u_{i}(x, t), i=1,2$ are the averaged horizontal velocities. $I$ denotes the projection of the channel occupied by the fluid onto the x-direction. $I=[0, L], \mathrm{L}$ is the length of the channel.

\subsection{Shear stresses}

- At the bottom the friction term is:

$$
\tau_{b}=c_{d} u_{2}\left|u_{2}\right|
$$

The values of the bottom drag coefficient $c_{d}$, usually used to account for bottom stresses in straits or estuaries range from $10^{-3}$ to $5.10^{-3}$. A typical value of $c_{d}=3.10^{-3}$, representing the drag along solid boundaries is chosen in this case.

- At the interface the friction term is:

$$
\tau_{I}=c_{I}\left|u_{2}-u_{1}\right|\left(u_{1}-u_{2}\right) .
$$

The friction term is expressed by means of the inter facial stress coefficient $c_{I}$. Values of $c_{I}$ obtained from laboratory experiments range from $4.10^{-4}$ to $2.6 \times 10^{-3}$, which are consistent with the value used in the literature [5] for numerical computation. $c_{I}$ was set equal to $10^{-3}$.

- At the surface the atmospheric wind stresses have not been taken into account since their effect is small in comparison with the tidal forcing, $\tau_{w}=0$.

- Since we neglect the y-direction of the velocity the Coriolis effects vanish.

The parameters used for the mean stratification are:

$$
\rho_{1}=1027 \mathrm{~kg} / \mathrm{m}^{3}, \rho_{2}=1029 \mathrm{~kg} / \mathrm{m}^{3}, g=9.81 \mathrm{~kg} / \mathrm{s}^{2} .
$$




\subsection{Bathymetry of the Strait}

$\frac{\partial Z_{f}}{\partial x}$ represent the bed slope of the channel. The Strait of Gibraltar lies between the Cap TrafalgarCap Spartel section at the western end and the point d'Europe-point Almina section at the eastern end. The main topographic features of the Strait are as follows (see figure 2):

- Tarifa Narrows: it is a deep, narrow and steep sided trough with a fairly flat bottom at about $800 \mathrm{~m}$. Its width varies between $20 \mathrm{~km}$ at Gibraltar and $14 \mathrm{~km}$ at point Cires. The distance from Gibraltar to Tarifa is $25 \mathrm{~km}$.

- Camarinal sill: it is the main bathymetric sill, lying $13 \mathrm{~km}$ west of Tarifa, with a maximum depth of $300 \mathrm{~m}$. It is the section where internal waves are generated.

- Tanger basin: it is a relatively deep reservoir $400 m$ lying between Spartel and Camarinal sills. It has an important influence on the outflowing Mediterranean water.

- Spartel sill: it is located $28 \mathrm{~km}$ west of Camarinal sill with a maximum depth of $350 \mathrm{~m}$.

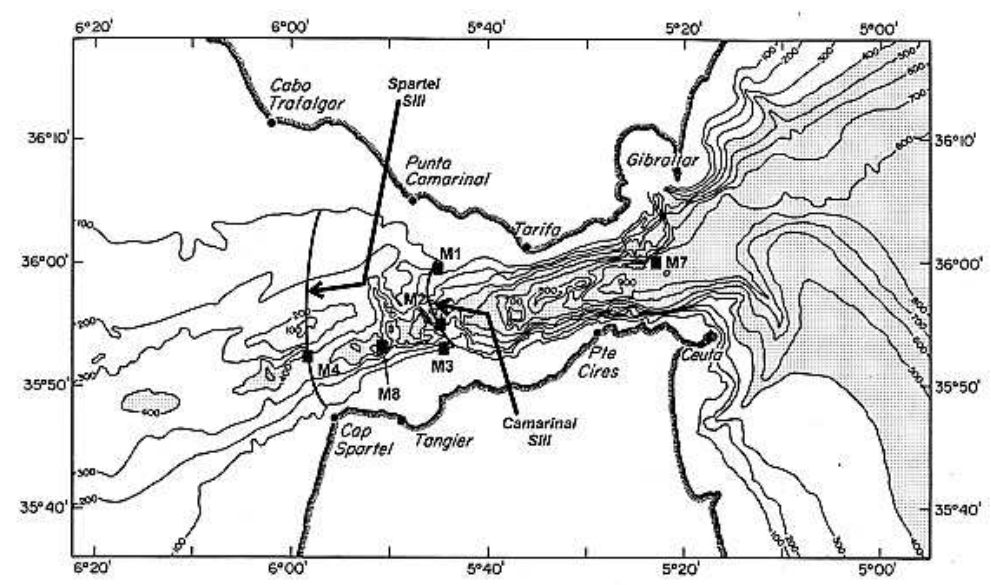

Figure 3: Bathymetry of the Strait of Gibraltar

\section{Finite volume formulation}

Equations (2.4) and (2.5) represent the same kind of equations that can be written as :

$$
\left\{\begin{array}{l}
\frac{\partial w}{\partial t}+\frac{\partial F}{\partial x}(w)=S, x \in I \subset I R, t \in[0, T] \\
w(x, 0)=w^{0}(x), x \in I \\
+ \text { Boundary Conditions, }
\end{array}\right.
$$


where:

$$
w(x, t)=\left(\begin{array}{c}
h(x, t) \\
h(x, t) u(x, t)
\end{array}\right), F(w)=\left(\begin{array}{c}
q \\
\frac{q^{2}}{h}+\frac{g h^{2}}{2}
\end{array}\right), S=\left(\begin{array}{c}
0 \\
R H S
\end{array}\right) .
$$

where $R H S$ could be $S_{1}$ or $S_{2}$ (see equations (2.6), (2.7)). In the context of finite volume schemes we are seeking for an approximate constant by cell solution. Let use the following notations:

$$
I_{i}=\left[x_{i-\frac{1}{2}}, x_{i+\frac{1}{2}}\left[, T_{n}=\left[t_{n}, t_{n+1}\left[, x_{i}=i \Delta x, \quad i \in N, t_{n+1}=t_{n}+\Delta t, \quad n \in N, \quad r=\frac{\Delta t}{\Delta x},\right.\right.\right.\right.
$$

the basic idea is to discretize the conservation law

$$
\int_{x_{i-\frac{1}{2}}}^{x_{i+\frac{1}{2}}} \int_{t_{n}}^{t_{n+1}}\left(\frac{\partial w}{\partial t}+\frac{\partial F(w)}{\partial x}\right) d t d x=\int_{x_{i-\frac{1}{2}}}^{x_{i+\frac{1}{2}}} \int_{t_{n}}^{t_{n+1}} S d t d x
$$

After integration the above equation becomes:

$$
\int_{x_{i-\frac{1}{2}}}^{x_{i+\frac{1}{2}}}\left(w\left(x, t_{n+1}\right)-w\left(x, t_{n}\right)\right) d x+\int_{t_{n}}^{t_{n+1}}\left(F\left(w\left(x_{i+\frac{1}{2}}, t\right)\right)-F\left(w\left(x_{i-\frac{1}{2}}, t\right)\right)\right) d t=\int_{x_{i-\frac{1}{2}}}^{x_{i+\frac{1}{2}}} \int_{t_{n}}^{t_{n+1}} S d t d x .
$$

If we denote

$$
w_{i}^{n} \approx \frac{1}{\Delta x} \int_{x_{i-\frac{1}{2}}}^{x_{i+\frac{1}{2}}} w\left(x, t_{n}\right) d x, I F_{i+\frac{1}{2}} \approx \frac{1}{\Delta t} \int_{t_{n}}^{t_{n+1}} F\left(w\left(x_{i+\frac{1}{2}}, t\right)\right) d t
$$

since we are seeking for an approximate constant by cell solution we can write $\int_{x_{i-\frac{1}{2}}}^{x_{i+\frac{1}{2}}} S d x \approx \Delta x \Delta t S_{i}^{n}$, then we get the following scheme:

$$
w_{i}^{n+1}=w_{i}^{n}-r\left(I F_{i+\frac{1}{2}}-I F_{i-\frac{1}{2}}\right)+\Delta t S_{i}^{n}
$$

Where $I F_{i+\frac{1}{2}}$ is the numerical flux. To determine the numerical flux, we apply the Roe's approximate Riemann solver [23], which is a linear wave decomposition. As the system is hyperbolic, the eigenvalues $\lambda_{1}=u+\sqrt{g h}$ and $\lambda_{2}=u-\sqrt{g h}$ of the Jacobian matrix

$$
A=\frac{\partial F}{\partial w}=\left(\begin{array}{cc}
0 & 1 \\
-u^{2}+g h & 2 u
\end{array}\right),
$$

correspond to the two characteristic speeds, and therefore their signs indicate the directions of propagation of information in the channel. Because of the system is hyperbolic there exists a 
regular matrix $R$ of eigenvectors of $A$ such that $A=R \Lambda R^{-1}$ where $\Lambda$ is a diagonal matrix of eigenvalues $\lambda_{i}$ of $A, \Lambda$ can be written as $\Lambda=\Lambda^{+}+\Lambda^{-}$where $\Lambda^{ \pm}=\operatorname{diag}\left( \pm \max \left( \pm \lambda_{i}, 0\right)\right)$. Let's define $A^{ \pm}=R \Lambda^{ \pm} R^{-1}$ then $A=A^{+}+A^{-}$and let's denote $|A|=A^{+}-A^{-}$. In order to construct a scheme taking into account these flow directions, an appropriate decomposition of the flux related to positive and negative propagation speeds is needed. The scheme has to be written as $[18,23]$ :

$$
I F_{i+\frac{1}{2}}=\frac{1}{2}\left(F\left({ }^{r} w\right)+F\left({ }^{l} w\right)\right)-|\bar{A}|\left({ }^{r} w-{ }^{l} w\right),
$$

where $\bar{A}$ is a constant matrix at every time level and for each pair of left and right states ${ }^{l, r} w$ satisfying the following conditions:

$$
1-F\left({ }^{r} w\right)-F\left({ }^{l} w\right)=\bar{A}\left({ }^{r} w,{ }^{l} w\right)\left({ }^{r} w-{ }^{l} w\right),
$$

2 - $\bar{A}\left({ }^{r} w{ }^{l} w\right)$ has real eigenvalues and a complete set of eigenvectors,

$$
3-\bar{A}(w, w)=A(w),
$$

$4-{ }^{r} w-{ }^{l} w=R\left({ }^{r} w,{ }^{l} w\right) \alpha=R((\bar{w})) \alpha$.

Property 1 ensures the conservation of the scheme, while the consistency with the original problem is guaranteed by property 2 and the hyperbolicity by property $3 . R$ is the matrix of eigenvectors, the $i^{\text {th }}$ element of $\alpha$ is the strength of the $i^{\text {th }}$ characteristic wave. The calculation of $\bar{A}$ is transferred to the calculation of $\bar{u}$ and $\bar{h}$, with the above requirements about the matrix $\bar{A}$. The solution is given by:

$$
\bar{h}=\frac{1}{2}\left({ }^{l} h+{ }^{r} h\right)
$$

where the Roe averaged state can readily be obtained as ([18], [23]):

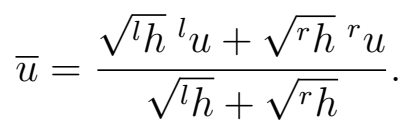

\subsection{Stability of the scheme}

The ratio $\frac{\Delta t}{\Delta x} \max _{p}\left|\bar{\lambda}_{p}^{ \pm}\right|$(where $\bar{\lambda}_{p}^{ \pm}=\frac{1}{2}\left(\lambda_{p} \pm\left|\lambda_{p}\right|\right)$ are the eigenvalues of $\bar{A}$ ) called more frequently the Courant number. It measures the fraction of a grid cell that information propagates through in one time step. For hyperbolic equations we typically use explicit methods and grids for which the Courant number is somewhat smaller than 1. Then the numerical scheme (3.3-3.4) must satisfy the following CFL (Courant, Friedrichs and Lewy) condition to be stable [17], [18], [23]:

$$
r \max _{p}\left|\bar{\lambda}_{p}^{ \pm}\right| \leq 1 .
$$




\subsection{Boundary conditions, initial conditions and source terms}

The major difficulties associated with the simulation of a two-layer flow trough the Strait of Gibraltar are:

\section{- Initial conditions :}

It will be assumed that the initial state corresponds to a steady state, but what is the meaning of the steady state in our situation?

The real answer is that some pseudo-steady state could only be imagined as the steady state would correspond to the time of the breach opening between the Atlantic Ocean and Mediterranean Sea some centuries ago. This pseudo-steady state can be either determined by assuming time-independent prescribed boundary conditions.

\section{- Open boundary conditions :}

The principal difficulty in modelling a limited region of an ocean basin associated with the correct specification of conditions along the open boundaries where the computational domain is artificially truncated. In our case the boundary condition on the free surface was taken in the formulation of the model. For the open boundary we have considered the condition of inflow driven by the tidal wave inside the Atlantic ocean. Inside the Mediterranean sea, the depth of the layers are set constant.

\section{- Source terms :}

The source terms of (2.4)-(2.5) are stiff, implementing them directly causes an instability as it was shown by Ambrosi [1] for a simple explicit discretization of source term integrated in the finite volume scheme for 1D Shallow Water model. An alternative approach is to employ a time-splitting in which one alternative between solving a system of conservation laws, with no source terms, and a system of ordinary differential equations modelling the source terms. In the simplest case this splitting takes the form ( $[11],[26])$ :

$$
w^{n+1}=L_{s}^{\Delta t} L_{f}^{\Delta t} w^{n},
$$

where $L_{f}^{\Delta t}$ represent the numerical solution operator for the conservation law:

$$
\frac{\partial w}{\partial t}+\frac{\partial F(w)}{\partial x}=0
$$

over the time $\Delta t$, and $L_{s}^{\Delta t}$ the numerical solution operator for the ODE:

$$
\frac{\partial w}{\partial t}=S(w)
$$




\section{Numerical results}

We have performed a numerical scheme based on the finite volume method to simulate the twolayer shallow water equations. The application considered concerns the Strait the of Gibraltar. The computational results obtained are compared to experiments realized by Kinder (CTD experiments 1985), and to the numerical results provided by Ouazar et Al. [22]. We recall that in [22], the authors used the method of characteristics (MOC), they neglected the secondary effects and have taken into account the geometry of the channel. In the present work we have considered only a large channel, we have included the shear stresses at the bottom and at the interface. The interface has been determined along $68 \mathrm{Km}$ of length from cap Spartel section to Gibraltar section. The figure 4 shows the location of the interface at time $t=6$ hour $s$ along the bed slop of the the Strait. The mesh size is chosen by considering bottom irregularities for the minimum spatial step $\Delta x$ and by satisfying the CFL criteria for the time step $\Delta t$, we take then $\Delta x=3.3 \mathrm{~km}$ and $\Delta t=70 \mathrm{~s}$.

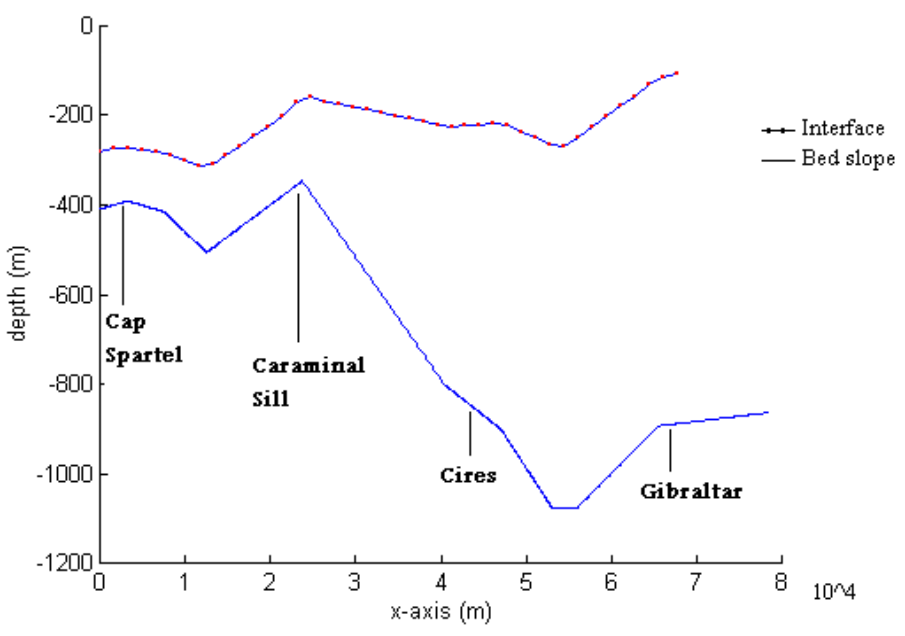

Figure 4: Interface location at time $\mathrm{t}=6$ hours.

Figure 5 shows a comparison between the numerical simulation of the interface and the experiments. The numerical simulation is in close agreement with Kinder's measurement along the longitudinal profile studied. However, some largest discrepancies west of the Cires section are observed. This can be explained by the fact that the bathymetry is not really known in this zone because of the presence of steep slopes and a moving bed creating some hydrodynamic instabilities.

Figure 6 represents the maximum and the minimum envelope curves corresponding to minimal and maximal depths for each node.

In Figure 7, the minimal interface depth is plotted at $t=6$ hours, the numerical simulation of the interface using method of characteristic and Kinder's measurements. The numerical simulation results obtained seems to be in close agreement with Kinder's measurements as well with numerical simulation using MOC method [22] along the longitudinal profile studied. 


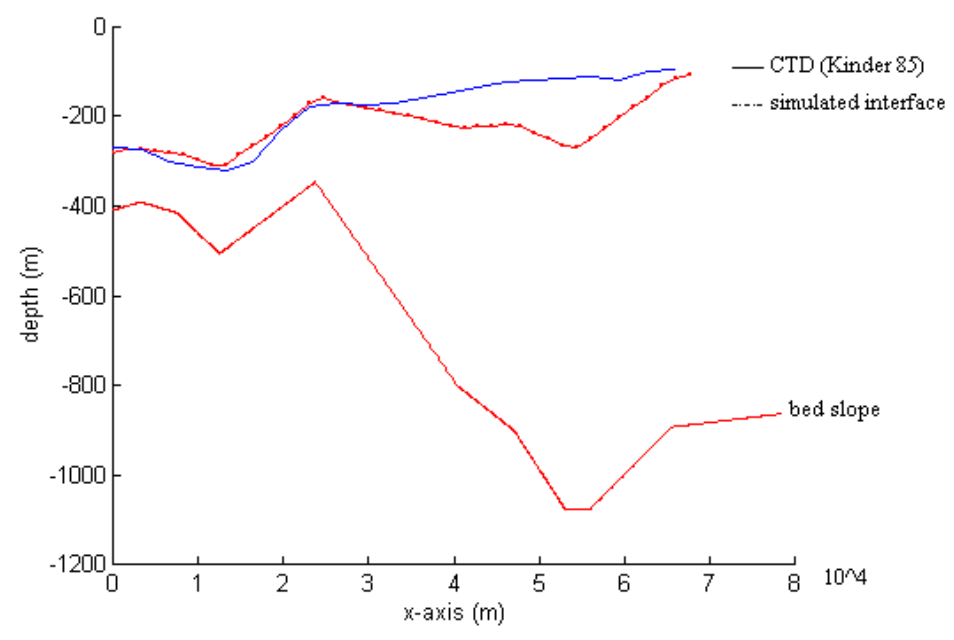

Figure 5: Comparison of numerical simulation and experiments.

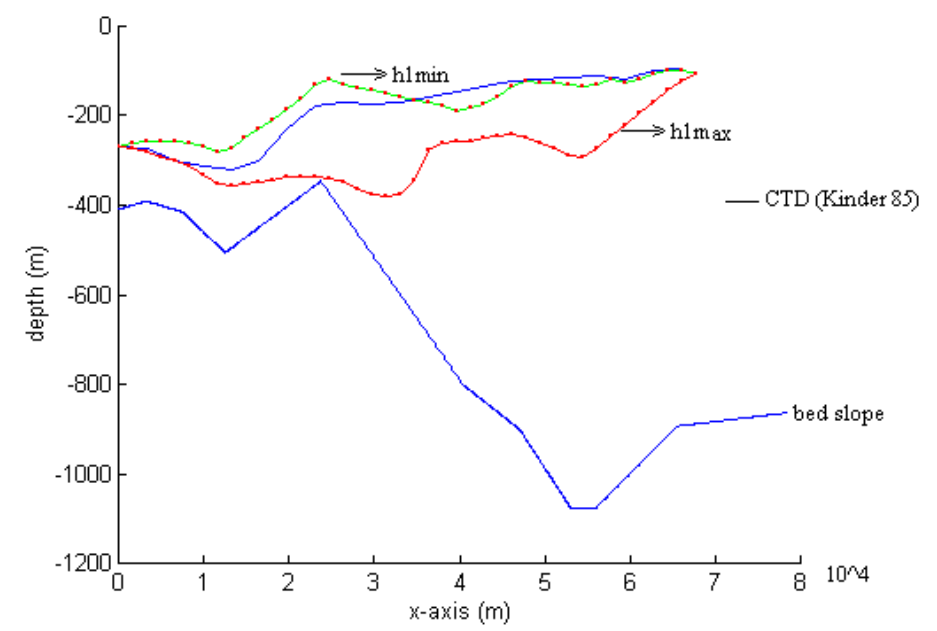

Figure 6: Maximum and minimum depths compared to Kinder's results. 


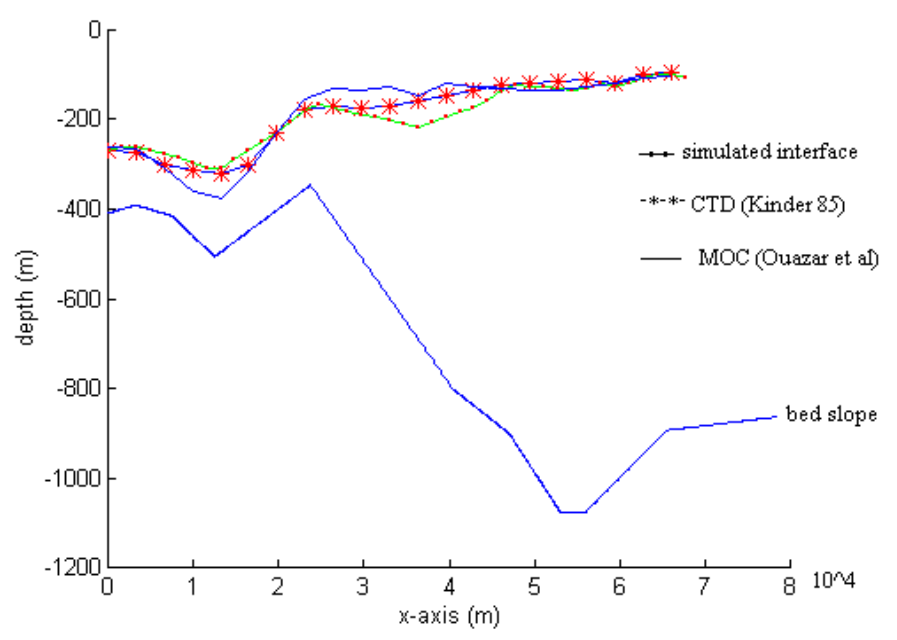

Figure 7: Minimum depth compared to the Kinder's measurements and to the method of characteristic (MOC).

\section{Concluding remarks}

Under certain plausible assumptions, the hydrodynamic equations governing a two-layered flow in the Strait of Gibraltar have been integrated numerically using the finite volume method based on Roe scheme approximation. Comparisons with published field results show a surprising close agreement for the location of the mean interface and its variability. The results being very encouraging, the authors are extending the simplified model by introducing $2 \mathrm{D}$ computational technique and taking into account the geometry of the channel and all the effects.

\section{References}

[1] D. Ambrosi. Approximation of shallow water equations by Riemann solvers. Inter. Journ. for Numerical Methods in Fluids,(1995) 20, 157-168.

[2] L. Armi, D. Farmer. The internal hydraulics of the Strait of Gibraltar and associated sills land narrows. Oceanologia Acta 8, (1985), 37-46.

[3] L. Armi, D. Farmer. The flow of Mediterranean water through the Strait of Gibraltar. Prog. Oceanogr., 21, (1988), 1-105.

[4] N. Benmansour. Resultats de la Compagne CTD sur le Lynch-2 Gibraltar Experiments. Rapport de mission, Ecole Mohammadia d'Ingenieurs, (1985).

[5] N. Benmansour. Boundary element solution to stratified shallow water wave equations (with application to the strait of Gibraltar). PHD Thesis, Wesses Institute of Technology, University of Portsmouth, (1993). 
[6] H. L. Bryden, T. H. Kinder. Gibraltar experiment. A plan for dynamics and kinematic investigations of strait mixing, exchange and turbulence. WHOI-86-29, (1986).

[7] H. L. Bryden, T. H. Kinder. The 1985 - 1986 Gibraltar Experiment : Data collection and preliminary results. Eos Trans. AGU, 68, 786-787, 793-795.

[8] H. L. Bryden, T. H. Kinder. Gibraltar experiment. Summary and initial results of Gibraltar experiments. WHOI-88-30, (1988).

[9] H. L. Bryden, T. H. Kinder. Measurements of the flow throught the Strait of Gibraltar. Proc. 1st Int. Conf. in Africa on Computer Methods and Water Resources, Vol.2 Computational Hydraulics, Ed. D. Ouazar et al, CMP and CMP and Spring-Verlag, (1988), 347-362.

[10] H. L. Bryden, H. M. Stommel. Limiting process that determines basic features of circulation in the Mediterranean sea. Oceanoligica Acta, 7 (1984), 286-296.

[11] M. Chakir, F. Benkhalodoun, L. Monthe, D. Ouazar, A. Belghit. A nine points finite volume Roe scheme for shallow water equations coupled to pollutant transport including source terms. Proc. of 1st Int. Symposium on Finite Volumes for Complex Applications, (1996), 625-633.

[12] L. Gonzalez Alonso. Liaison fixe à travers le détroit de Gibraltar: la solution ferroviaire = fixed connexion between Gibraltar channel: the railway solution. Rail international ISSN 1022-4076 CODEN RAIIAF, (1984), No. 8-9, 30-31.

[13] M. Gonzalez, M. Seaid. Finite element modified method of characterstics for shallow water flows: application to the strait of Gibraltar, Progress in Industrial Mathematics, 8 (2005), 512-522.

[14] H. Lacombe, C. Richez. The regime of the strait of Gibraltar. Proc. Hydrodynamics of SemiEnclosed Seas. Ed. J.C.J. Nihoul, Elservier, Amsterdam, (1982), 13-74.

[15] H. Lacombe, C. Richez. Hydrography and currents in the strait Gibraltar. Sea Straits Research Atlas SSR-3. National Ocean Research and development Activity, NSTL Mississippi 39529.

[16] P.E. La Violette, T.H. Kinder, D.W. Green, Measurements of internal waves in the Strait of Gibraltar using a shore-based radar. Tech. Rep. 118,13 pp., Nav. Ocean Res. and Develop. Activ., Natl. Space Technol. Lab., Bay St Louis, Miss. (1986)

[17] R. J. Leveque. A study of numerical methods for hyperbolic conservation laws with stiff source terms. Journal of Computational Physics, 86 (1979), 187-210.

[18] R. J. Leveque. Numerical methods for conservation laws. Lectures in Mathematics ETH Zurich, Birhauser Verlag, 1992. 
[19] F. Madelain. Influence de la Topographie du fond sur l'écoulement Méditerraneen entre le Détroit de Gibraltar et le Cap Saint-Vincent. Cahiers Océanographiques, 22 (1970), No 1.

[20] M. Mahboub. Modèle Numérique Eléments finis du Détroit de Gibraltar, EMI, Graduation Thesis, Rabat, (1988).

[21] L. Moutya. Modèle Unidimensionnel Bi-couche du Détroit de Gibraltar, pp VII. 11-1/11-13, Proc. 1er Colloque Maghrébin sur les Modèles Numériques de l’Ingénieur, Algeria, (1987).

[22] D. Ouazar, N. Benmansour, C. A. Brebbia. Towards a numerical model of strait of Gibraltar, Software for hydraulics, hydrology and hydrodynamics, volume, No. 3, (1989).

[23] P. L. Roe. Approximate Riemann solvers, parameter vectors, and difference scheme. Journal of computational physics 43 (1981), 357-372.

[24] A. Taik. Modélisation et analyse numérique des écoulements à surface libre par un schema volumes finis et par la méthode des étéments finis. thèse de Doctorat d'Etat, (2002), EMIRabat, Maroc.

[25] M. Taik. Projet de liaison fixe à travers le Détroit de Gibraltar: Projet de pont et navigation maritime. International navigation congress No 28, Seville , Espagne (22/05/1994) 1994, No. 83-84, 140-144, ISSN 0374-1001.

[26] H. G. Yee. A Class of high-resolution explicit and implicit shock-capturing methods. NASA Technical Memorandum 101088, 1989. 